\title{
EXACT TRAVELING WAVE SOLUTIONS OF A GENERALIZED KAWAHARA EQUATION
}

\author{
Elena V. Nikolova ${ }^{1 *}$, Zlatinka I. Dimitrova ${ }^{2}$ \\ ${ }^{1}$ Institute of Mechanics, Bulgarian Academy of Sciences, 4, Acad. G. Bonchev \\ Str. BG-1113 Sofia, Bulgaria \\ ${ }^{2}$ Institute of Solid State Physics, Bulgarian Academy of Sciences, 72, \\ Tsarigradsko Chaussee, BG-1784 Sofia, Bulgaria
}

[Received: 13 February 2019. Accepted: 13 May 2019]

doi: 10.7546/JTAM.49.19.02.03

\begin{abstract}
We apply the modified method of simplest equation for obtaining exact solutions of nonlinear partial differential equations (PDEs) from the kind of the generalized Kawahara equation (gKE). The ordinary differential equation of Abel of first kind is used as the simplest equation and two exact solutions of the studied equation are obtained. These solutions are expressed by the special function discussed in [1]. Particular cases of one of the obtained solutions are visualized. For these cases the special function is reduced to elementary functions and the corresponding solutions describe a kink and a solitary wave.
\end{abstract}

KEY WORDS: Kawahara equation, exact analytical solution, modified method of simplest equation, kink and solitary waves.

\section{INTRODUCTION}

Numerous models of natural and social systems are based on differential equations [2-11]. Exact solutions of these model equations are important as they give us useful information for understanding the states and processes in the modeled systems. In addition the exact solutions can be used to test computer programs for numerical simulations. Often the model equations are nonlinear partial differential equations and usually these model equations have traveling-wave solutions that are studied very intensively [12-22]. Powerful methods exist for obtaining exact traveling-wave solutions of nonlinear partial differential equations, e.g. the method of inverse scattering transform or the method of Hirota [14,23,24]. These methods are appropriate for the case of integrable nonlinear PDEs. Other approaches for obtaining exact special solutions of nonlintegrable nonlinear PDEs have been developed in the recent years (for examples see [25-29]). Below we shall discuss the method of simplest equation

\footnotetext{
${ }^{*}$ Corresponding author e-mail: elena@imbm.bas.bg
} 
and our focus will be on a version of the method called modified method of simplest equation [1,30-34]. The method of simplest equation is based on a procedure analogous to the first step of the test for the Painleve property [35-37]. In the version of the method called modified method of simplest equation [38-40], [41-43] this procedure is substituted by the concept for the balance equations. We shall mention below the possibility of use of more than one simplest equation for obtaining exact analytical solutions of the studied nonlinear partial differential equation $[44,45]$. In addition the modified method of simplest equation may lead to more than one balance equation. The modified method of simplest equation is already applied to obtain exact traveling wave solutions of the generalized Kuramoto-Sivashinsky equation, the reaction-diffusion equation, the reaction-telegraph equation [33], the generalized Swift-Hohenberg equation, the generalized Rayleigh equation [34], the generalized Fisher equation, the generalized Huxley equation [38], the generalized Degasperis-Procesi equation and b-equation [40], the extended Korteweg-de Vries equation [41-43, 46-50], etc.

\section{Methodology and Problem Formulation}

We are going to apply the modified method of simplest equation. The last version of the methodology used by our research group is based on the possibility of use of more than one simplest equation (see [51] for application of the method based on two simplest equations). The steps of the methodology are as follows:

1. By means of appropriate ansätze (e.g, one or several traveling-wave ansätze such as $\xi=\alpha x+\beta t ; \zeta=\gamma x+\delta t, \ldots)$ the studied nonlinear PDEs becomes a differential equation $D E$, containing derivatives of one or several functions

$$
D E\left[a(\xi), a_{\xi}, a_{\xi \xi}, \ldots, b(\zeta), b_{\zeta}, b_{\zeta \zeta}, \ldots\right]=0 .
$$

2. The functions $a(\xi), b(\zeta)$, etc., are searched as some function of another functions, e.g., $g(\xi), \phi(\zeta)$, etc., i.e.

$$
a(\xi)=G[g(\xi)] ; \quad b(\zeta)=F[\phi(\zeta)] ; \ldots
$$

3. Note that the kind of the functions $F, G, \ldots$ is not prescribed. One uses a finite-series relationship, e.g.,

$$
a(\xi)=\sum_{\mu_{1}=-\nu_{1}}^{\nu_{2}} q_{\mu_{1}}[g(\xi)]^{\mu_{1}} ; \quad b(\zeta)=\sum_{\mu_{2}=-\nu_{3}}^{\nu_{4}} r_{\mu_{2}}[\phi(\zeta)]^{\mu_{2}}, \ldots
$$


where $q_{\mu_{1}}, r_{\mu_{2}}, \ldots$ are coefficients. The functions $g(\xi), \phi(\zeta)$ are solutions of simpler ordinary differential equations called simplest equations. The idea to use of more than one simplest equation can be traced back at least to the early articles of Martinov and Vitanov [52-54].

4. After the substitution Eq. (2) in Eq. (1) we obtain a polynomial containing $g(\xi), \phi(\zeta), \ldots$ Then a balance procedure is applied. It has to ensure that all the coefficients of the obtained polynomial of $g(\xi)$ and $\phi(\zeta)$ contain more than one term. This procedure leads to one or more balance equations relating some of the parameters of the solved equation and some of the parameters of the solution. Note that the coefficients of all powers of the polynomials have to be balanced (and not only the coefficient of the largest power). This kind of balance may require more than one balance equation.

5. Eqs. (2) represent a candidate for solution of Eq. (1) if all coefficients of the obtained polynomial of are equal to 0 . This condition leads to a system of nonlinear algebraic equations for the coefficients of the solved nonlinear PDE and for the coefficients of the solution. Any nontrivial solution of this algebraic system leads to a solution of the studied nonlinear PDE.

We shall apply a particular case of the above methodology (based on one simplest equation [55]) to the generalized Kawahara equation [56-59]

$$
\gamma u_{t}+\alpha u^{n} u_{x}+\beta u_{x x x}-\delta u_{x x x x x}=0,
$$

where $\gamma, \alpha, \beta$ and $\delta$ are parameters and $n$ is a non-negative natural number. For the cases $n=1$ and $n=2 \mathrm{Eq}$. (4) is reduced to the Kawahara equation (KE) and the modified Kawahara equation (mKE). The Kawahara equation has numerous applications, e.g., for modeling shallow water waves on water surface or under the ice cover $[60,61]$. We introduce the ansatz of a traveling-wave variable, i.e. $\zeta=\theta x+\nu t$ in Eq. (4) and we search for a solution of Eq. (4) of the kind:

$$
u=u(\zeta)=\sum_{q=0}^{r} a_{q} g^{q} ; \quad \frac{d g}{d \zeta}=\sum_{m=0}^{l} b_{m} g^{m},
$$

where $a_{q}$ and $b_{m}$ are parameters, and $g(\zeta)$ is a solution of simplest equation. Next step is to obtain the balance equation by balancing the powers of $g$ arising from the terms in Eq. (4). The balance equation is $q n=4 m-4$. In this study we shall use the ordinary differential equation of Abel of first kind as a simplest equation

$$
\frac{d g}{d \zeta}=b_{0}+b_{1} g+b_{2} g^{2}+b_{3} g^{3} .
$$




\section{Several Solutions of Equations of the Class of Eq. (4)}

\subsection{CASE $n=1$}

Let us firstly consider the case $n=1$. Then Eq.(4) becomes

$$
\gamma u_{t}+\alpha u u_{x}+\beta u_{x x x}-\delta u_{x x x x x}=0 .
$$

In this case the balance equation becomes $q=4 m-4$. For the case of use of Abel equation of first kind as a simplest equation the solution of Eq. (7) can be presented as

$$
u(\zeta)=a_{0}+a_{1} g+a_{2} g^{2}+a_{3} g^{3}+a_{4} g^{4}+a_{5} g^{5}+a_{6} g^{6}+a_{7} g^{7}+a_{8} g^{8} .
$$

The solution of the differential equation of Abel can be written as

$$
\left(\frac{d g}{d \zeta}\right)^{2}=c_{0}+c_{1} g+c_{2} g^{2}+c_{3} g^{3}+c_{4} g^{4}+c_{5} g^{5}+c_{6} g^{6},
$$

where

$$
\begin{array}{llll}
c_{0}=b_{0}^{2} ; & c_{1}=2 b_{0} b_{1} ; & c_{2}=2 b_{0} b_{2}+b_{1}^{2} ; & c_{3}=2 b_{0} b_{3}+2 b_{1} b_{2} ; \\
c_{4}=2 b_{1} b_{3}+b_{2}^{2} ; & c_{5}=2 b_{2} b_{3} ; & c_{6}=b_{3}^{2}
\end{array}
$$

and its solution is given by the special function $V_{c_{0}, c_{1}, c_{2}, c_{3}, c_{4}, c_{5}, c_{6}}(\zeta ; 1,2,6)$ [1]. In general the special function $V_{c_{0}, c_{1} ; \ldots, c_{m}}(\zeta ; k, l, m)$ is the solution of the ordinary differential equation

$$
\left(\frac{d^{k} g}{d \zeta^{k}}\right)^{l}=\sum_{j=0}^{m} c_{j} g^{j} .
$$

Then the solution of Eq. (9) is

$$
g(\zeta)=V_{b_{0}^{2}}, 2 b_{0} b_{1}, 2 b_{0} b_{2}+b_{1}^{2}, 2 b_{0} b_{3}+2 b_{1} b_{2}, 2 b_{1} b_{3}+b_{2}^{2}, 2 b_{2} b_{3}, b_{3}^{2}(\zeta ; 1,2,6) .
$$

The relationships among coefficients of the solution and coefficients of the model are obtained by solving a system of eighteen algebraic equations. This system is very large and we shall not write it here. The process of solution of the algebraic system leads to the following relationships (we note that below the division in all ratios is made and thus they are written as real numbers):

$$
\begin{aligned}
a_{0}= & \left(-27 \times 10^{2} \theta^{5} \delta^{2} b_{2}^{10}-15 \times 10^{2} \theta^{5} \delta^{2} b_{2}^{8} M_{2}-260 \theta b_{3}^{4} b_{2}^{2} \beta^{2}+\right. \\
& +38.5 \times 10^{2} \theta^{5} \delta^{2} b_{2}^{6} M_{2}^{2}+0.08 \theta^{2} b_{2}^{4} M_{1}^{3} \delta^{-1}-3.5 \times 10^{-6} \theta b_{2}^{2} M_{1}^{4} \delta^{-2}
\end{aligned}
$$




$$
\begin{aligned}
& +2 \theta^{3} b_{2}^{4} M_{1}^{2} M_{2}-0.03 \theta^{3} M_{1}^{2} b_{2}^{2} M_{2}^{2}-35 \times 10^{2} \theta^{3} b_{3}^{2} \delta b_{2}^{4} \beta M_{2} \\
& +70 \theta^{2} b_{3}^{2} b_{2}^{4} \beta M_{1}+6.5 \times 10^{-6} M_{1}^{5} \delta^{-3}-13 \times 10^{2} \theta^{3} b_{3}^{2} \delta b_{2}^{6} \beta \\
& +225 \theta^{4} \delta b_{2}^{8} M_{1}+6 \times 10^{2} \theta^{4} \delta b_{2}^{6} M_{1} M_{2}-42.5 \times 10^{2} \theta^{3} b_{2}^{6} M_{1}^{2} \\
& +1.6 \theta^{5} \delta^{2} b_{2}^{4} M_{2}^{3}+2 b_{3}^{4} M_{1} \gamma \nu \theta^{-1}-42.5 b_{3}^{4} \delta b_{2}^{2} \gamma-1.3 \theta b_{3}^{2} b_{2}^{2} \beta M_{1}^{2} \delta^{-1} \\
& +115 \theta^{4} \delta b_{2}^{4} M_{1} M_{2}^{2}+6.5 \theta^{2} b_{3}^{2} b_{2}^{2} \beta M_{1} M_{2}+1.6 b_{3}^{4} M_{1} \beta^{2} \delta^{-1} \\
& \left.+3 \times 10^{-3} b_{3}^{2} M_{1}^{3} \beta \delta^{-2}-0.06 \theta^{2} b_{2}^{2} M_{1}^{3} M_{2} \delta^{-1}\right) / \theta b_{3}^{4} \delta \alpha M_{3} .
\end{aligned}
$$

We obtain for the parameter $a_{1}$

$$
\begin{aligned}
a_{1}= & -0.3 \gamma \nu \delta-7.8 \times 10^{-5} \theta b_{3}^{2} \beta M_{1}^{2} \delta^{-1}+1.2 \theta^{3} b_{3}^{2} \beta \delta b_{2}^{4} \\
& +0.3 \theta^{3} b_{3}^{2} \beta b_{2}^{2} \delta M_{2}-1.7 \times 10^{-8} \theta M_{1}^{4} \delta^{-2}+1.8 \times 10^{-4} \theta^{2} b_{2}^{2} M_{1}^{3} \delta^{-1} \\
& -10^{-4} \theta^{3} b_{2}^{4} M_{1}^{2}-0.2 \theta b_{3}^{4} \beta^{2}+0.8 \theta^{5} \delta^{2} b_{2}^{8}-0.2 \theta^{2} b_{3}^{2} \beta b_{2}^{2} M_{1} \\
& +0.1 \theta^{4} \delta b_{2}^{4} M_{1} M_{2}+15 \theta^{5} \delta^{2} b_{2}^{4} M_{2}^{2}+0.04 \theta^{4} \delta b_{2}^{6} M_{1}^{2} \\
& +8 \times 10^{-4} \theta^{3} b_{2}^{2} M_{1}^{2} M_{2}+0.08 \theta^{4} \delta M_{1} b_{2}^{2} M_{2}^{2}+15 \theta^{5} \delta^{2} b_{2}^{6} M_{2} \\
& +\left(-7.4 \times 10^{3} \theta^{5} \delta^{2} b_{2}^{10}-4 \times 10^{2} \theta^{5} \delta^{2} b_{2}^{8} M_{2}-70 \theta b_{3}^{4} b_{2}^{2} \beta^{2}\right. \\
& +10^{4} \theta^{5} \delta^{2} b_{2}^{6} M_{2}^{2}+0.02 \theta^{2} b_{2}^{4} M_{1}^{3} \delta^{-1}-9.6 \times 10^{-5} \theta b_{2}^{2} M_{1}^{4} \delta^{2} \\
& +0.5 \theta^{3} b_{2}^{4} M_{1}^{2} M_{2}-8 \times 10^{-3} \theta^{3} M_{1}^{2} b_{2}^{2} M_{2}^{2}-952 \theta^{3} b_{3}^{2} \delta b_{2}^{4} \beta M_{2} \\
& +19 \theta^{2} b_{3}^{2} b_{2}^{4} \beta M_{1}+1.7 \times 10^{-7} M_{1}^{5} \delta^{3}+16.7 \theta^{4} \delta b 2^{6} M_{1} M_{2} \\
& -369 \theta^{3} b_{3}^{2} \delta b_{2}^{6} \beta+61 \theta^{4} \delta b_{2}^{8} M_{1}+0.18 \theta^{3} b_{2}^{6} M_{1}^{2}-4.8 \theta^{5} \delta^{2} b_{2}^{4} M_{2}^{3} \\
& +2.7 b_{3}^{4} M_{1} \gamma \nu \theta^{-1}-10^{-4} b_{3}^{4} \delta b_{2}^{2} \gamma \nu^{-2}-0.3 \theta b_{3}^{2} b_{2}^{2} \beta M_{1}^{2} \delta^{-1} \\
& +31.7 \theta^{4} \delta b_{2}^{4} M_{1} M_{2}^{2}+1.7 \theta^{2} b_{3}^{2} b_{2}^{2} \beta M_{1} M_{2}+0.2 b_{3}^{4} M_{1} \beta^{2} \delta^{-1} \\
& \left.\left.+8 \times 10^{-4} b_{3}^{2} M_{1}^{3} \beta \delta^{-2}-2.10^{-3} \theta^{2} b_{2}^{2} M_{1}^{3} M_{2} \delta^{-1}\right) / M_{3}\right) / \theta b_{3}^{3} \delta \alpha b_{2} ; \\
a_{2} & \theta^{2}\left(109.6 \theta^{2} \delta b_{2}^{4} M_{2}-0.6 b_{3}^{2} \beta M_{1} \delta^{-1} \theta^{-1}-0.4 b_{3}^{2} \beta b_{2}^{2}-1.6 \theta b_{2}^{4} M_{1}\right. \\
& +22 \theta^{2} \delta b_{2}^{6}+6.1 \theta^{2} \delta b_{2}^{2} M_{2}^{2}+0.1 b_{2}^{2} M_{1}^{2} \delta^{-1}+10^{-3} M_{1}^{3} \theta^{-1} \delta^{-2} \\
& \left.+8.4 \theta b_{2}^{2} M_{1} M_{2}\right) / b_{3}^{2} \alpha ; \\
a_{3} & \theta^{2}\left(1.1 \theta M_{1} b_{2} M_{2}-11.5 \beta b_{3}^{2} b_{2}+0.1 b_{2} M_{1}^{2} \delta^{-1}\right. \\
& \left.-3.1 \theta^{2} \delta b_{2}^{5}+120 \theta^{2} \delta b_{2}^{3} M_{2}+3 \theta b_{2}^{3} M_{1}\right) / b_{3} \alpha ;
\end{aligned}
$$$$
\text { (15) }
$$ 


$$
\begin{aligned}
\text { (17) } \quad a_{4}= & \theta^{2}\left(2.6 \times 10^{3} \theta^{2} \delta b_{2}^{2} M_{2}+2.9 \times 10^{3} \theta b_{2}^{2} M_{1}-86 \beta b_{3}^{2}+3.2 \theta^{2} \delta b_{2}^{4}\right. \\
& \left.+1.3 \delta^{-1} M_{1}^{2}\right) / \alpha ; \\
\text { (18) } \quad a_{5}= & \theta^{4} \delta b_{3}\left(1.6 \times 10^{3} b_{2} M_{2}+6 \times 10^{2} b_{2} M_{1} \delta^{-1} \theta^{-1}+2.3 \times 10^{4} b_{2}^{3}\right) / \alpha ; \\
\text { (19) } \quad a_{6}= & \theta^{4} b_{3}^{2} \delta\left(6.6 \times 10^{4} b_{2}^{2}+3.4 \times 10^{2} M_{1} \delta^{-1} \theta^{-1}\right) / \alpha ; \\
\text { (20) } \quad a_{7}= & 7 \times 10^{4} \delta \theta^{4} b_{3}^{3} b_{2} / \alpha ; \\
\text { (21) } \quad a_{8}= & 2.6 \times 10^{4} \delta \theta^{4} b_{3}^{4} / \alpha ; \\
\text { (22) } \quad b_{0}= & 4.10^{-2} b_{2} M_{2} / b_{3}^{2} ; \quad b_{1}=6 \times 10^{-3} M_{1} / \delta b_{3} \theta
\end{aligned}
$$

where

$$
\begin{aligned}
& M_{1}=52 \delta b_{2}^{2} \theta+6 \sqrt{13 \beta \delta} b_{3}, \\
& M_{2}=2 b_{2}^{2}+0,06 M_{1} / \delta \theta \\
& M_{3}=-4358 b_{2}^{2}+10,31 M_{1} / \delta \theta
\end{aligned}
$$

and $b_{2}$ and $b_{3}$ are free parameters. Thus the solution of Eq. (7) becomes

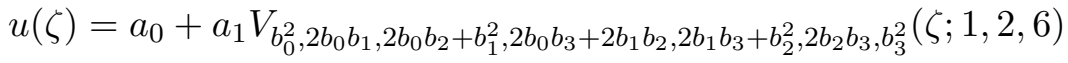

$$
\begin{aligned}
& +a_{2} V_{b_{0}^{2}}^{2}, 2 b_{0} b_{1}, 2 b_{0} b_{2}+b_{1}^{2}, 2 b_{0} b_{3}+2 b_{1} b_{2}, 2 b_{1} b_{3}+b_{2}^{2}, 2 b_{2} b_{3}, b_{3}^{2}(\zeta ; 1,2,6) \\
& +a_{3} V_{b_{0}^{2}}^{3}, 2 b_{0} b_{1}, 2 b_{0} b_{2}+b_{1}^{2}, 2 b_{0} b_{3}+2 b_{1} b_{2}, 2 b_{1} b_{3}+b_{2}^{2}, 2 b_{2} b_{3}, b_{3}^{2}(\zeta ; 1,2,6) \\
& +a_{4} V_{b_{0}^{2}}^{4}, 2 b_{0} b_{1}, 2 b_{0} b_{2}+b_{1}^{2}, 2 b_{0} b_{3}+2 b_{1} b_{2}, 2 b_{1} b_{3}+b_{2}^{2}, 2 b_{2} b_{3}, b_{3}^{2}(\zeta ; 1,2,6) \\
& +a_{5} V_{b_{0}^{2}, 2 b_{0} b_{1}, 2 b_{0} b_{2}+b_{1}^{2}, 2 b_{0} b_{3}+2 b_{1} b_{2}, 2 b_{1} b_{3}+b_{2}^{2}, 2 b_{2} b_{3}, b_{3}^{2}}(\zeta ; 1,2,6)+ \\
& +a_{6} V_{b_{0}^{2}, 2 b_{0} b_{1}, 2 b_{0} b_{2}+b_{1}^{2}, 2 b_{0} b_{3}+2 b_{1} b_{2}, 2 b_{1} b_{3}+b_{2}^{2}, 2 b_{2} b_{3}, b_{3}^{2}}(\zeta ; 1,2,6)+ \\
& +a_{7} V_{b_{0}^{2}}^{7}, 2 b_{0} b_{1}, 2 b_{0} b_{2}+b_{1}^{2}, 2 b_{0} b_{3}+2 b_{1} b_{2}, 2 b_{1} b_{3}+b_{2}^{2}, 2 b_{2} b_{3}, b_{3}^{2}(\zeta ; 1,2,6) \\
& +a_{8} V_{b_{0}^{2}}^{8}, 2 b_{0} b_{1}, 2 b_{0} b_{2}+b_{1}^{2}, 2 b_{0} b_{3}+2 b_{1} b_{2}, 2 b_{1} b_{3}+b_{2}^{2}, 2 b_{2} b_{3}, b_{3}^{2}(\zeta ; 1,2,6)
\end{aligned}
$$

We can obtain a particular case of this solution expressed by elementary functions for the special case $b_{0}=\frac{b_{2}}{3 b_{3}}\left(b_{1}-\frac{2 b_{2}^{2}}{9 b_{3}}\right)$. Then the Abel equation has the following solution:

$$
\begin{aligned}
g(\zeta) & =V_{b_{0}^{2}, 2 b_{0} b_{1}, 2 b_{0} b_{2}+b_{1}^{2}, 2 b_{0} b_{3}+2 b_{1} b_{2}, 2 b_{1} b_{3}+b_{2}^{2}, 2 b_{2} b_{3}, b_{3}^{2}(\zeta ; 1,2,6)} \\
& =\frac{\exp \left[\left(b_{1}-\frac{b_{2}^{2}}{3 b_{3}}\right) \zeta\right]}{\sqrt{C^{*}-b_{3} \exp \left[2\left(b_{1}-\frac{b_{2}^{2}}{3 b_{3}}\right) \zeta\right]}}-\frac{b_{2}}{3 b_{3}}
\end{aligned}
$$


where $C^{*}$ is a constant of integration. The substitution of Eqs. (13)-(25) in Eq.(26) leads to a particular case of the solution (26) expressed by elementary functions.

\subsection{CASE $n=2$}

Let us now consider the case $n=2$. Then Eq. (4) is reduced to the mKE in the form

$$
\gamma u_{t}+\alpha u^{2} u_{x}+\beta u_{x x x}-\delta u_{x x x x x}=0 .
$$

In this case the balance equation becomes $q=2 m-2$ and we search for the solution of Eq. (28) in the form

$$
u(\zeta)=a_{0}+a_{1} g+a_{2} g^{2}+a_{3} g^{3}+a_{4} g^{4},
$$

where $g(\zeta)$ is a solution of the Abel differential equation of first kind (Eq. (9)). The relationships among the coefficients of the solution and the coefficients of the model are derived by solving a system of fourteen algebraic equations. The process of solution of the algebraic system leads to the following relationships:

$$
\begin{aligned}
\text { (30) } a_{0} & =\sqrt{10 \alpha \delta}\left(-0.03 \theta^{2} b_{2}^{4} \delta L_{2}+0.03 \theta^{2} b_{2}^{4} \delta L_{2}^{2}+0.01 \theta^{2} \delta b_{2}^{4}\right. \\
& \left.+0.03 \theta^{2} b_{2}^{2} \delta\left(3 b_{2}^{2} L_{2}-2 b_{2}^{2}\right)-0.002 \sqrt{60 L_{4}} b_{2}^{4} \theta^{2} \beta L_{1}^{-2}\right) / \sqrt{L_{4}} b_{2}^{4} \theta^{2} \alpha \delta \\
\text { (31) } a_{1}= & \sqrt{10 \alpha \delta} \sqrt[4]{2.4} L_{1}\left(2.4 b_{2}^{3} L_{2}-0.3 b_{2}^{3}+0.5 b 2\left(3 b_{2}^{2} L_{2}-2 b 2^{2}\right)\right) / \sqrt[4]{L_{4}} b_{2}^{2} \alpha \\
\text { (32) } a_{2} & =8 \theta^{2} \sqrt{10 \alpha \delta}\left(b_{2}^{2}+b_{2}^{2} L_{2}\right) / \alpha \\
\text { (33) } a_{3} & =7.1 b_{2}^{3} \theta^{2} \sqrt[4]{540 L_{4}} \sqrt{10 \alpha \delta} / \alpha L_{1} \\
\text { (34) } a_{4} & =3.6 \theta^{4} \sqrt{10 \alpha \delta} \sqrt[4]{L_{4}} / \alpha L_{1} \\
\text { (35) } b_{0} & =0.004 \sqrt{60} L_{1}^{2}\left(3 b_{2}^{2} L_{2}-2 b_{2}^{2}\right) / b_{2}^{3} \theta^{2} \sqrt{L_{4}} \\
\text { (36) } b_{1} & =0.25 \sqrt[4]{2,4} L_{1} L_{2} / \theta \sqrt{L_{4}} \\
\text { (37) } b_{3} & =0.2 \sqrt[4]{540} \sqrt{L_{4}} / L_{1}
\end{aligned}
$$

where

$$
\begin{array}{ll}
L_{1}=\beta^{2} \theta+10 \gamma \nu \delta, & L_{2}=-2+\frac{1}{3} \sqrt{51} \\
L_{3}=-943+132 \sqrt{51}, & L_{4}=\theta \delta^{2} L_{3} L_{1}^{3}
\end{array}
$$

and $b_{2}$ is a free parameter. Thus the solution of Eq. (28) becomes

$$
u(\zeta)=a_{0}+a_{1} V_{b_{0}^{2}, 2 b_{0} b_{1}, 2 b_{0} b_{2}+b_{1}^{2}, 2 b_{0} b_{3}+2 b_{1} b_{2}, 2 b_{1} b_{3}+b_{2}^{2}, 2 b_{2} b_{3}, b_{3}^{2}}(\zeta ; 1,2,6)
$$




$$
\begin{aligned}
& +a_{2} V_{b_{0}^{2}, 2 b_{0} b_{1}, 2 b_{0} b_{2}+b_{1}^{2}, 2 b_{0} b_{3}+2 b_{1} b_{2}, 2 b_{1} b_{3}+b_{2}^{2}, 2 b_{2} b_{3}, b_{3}^{2}}(\zeta ; 1,2,6) \\
& +a_{3} V_{b_{0}^{2}, 2 b_{0} b_{1}, 2 b_{0} b_{2}+b_{1}^{2}, 2 b_{0} b_{3}+2 b_{1} b_{2}, 2 b_{1} b_{3}+b_{2}^{2}, 2 b_{2} b_{3}, b_{3}^{2}}^{3}(\zeta ; 1,2,6) \\
& +a_{4} V_{b_{0}^{2}, 2 b_{0} b_{1}, 2 b_{0} b_{2}+b_{1}^{2}, 2 b_{0} b_{3}+2 b_{1} b_{2}, 2 b_{1} b_{3}+b_{2}^{2}, 2 b_{2} b_{3}, b_{3}^{2}}^{4}(\zeta ; 1,2,6)
\end{aligned}
$$

For the special case $b_{0}=\frac{b_{2}}{3 b_{3}}\left(b_{1}-\frac{2 b_{2}^{2}}{9 b_{3}}\right)$ the Abel equation has the solution given by (27) and then the solution given by Eq. (40) can be expressed by elementary functions.

\section{Discussion}

Figures 1 and 2 show two kinds of waves obtained when the solution (26) of Kawahara equation can be expressed by elementary functions (27). These two kinds of waves are: solitary wave (Fig. 1) and kink wave (Fig. 2) The waves are obtained by

\section{Solitary}

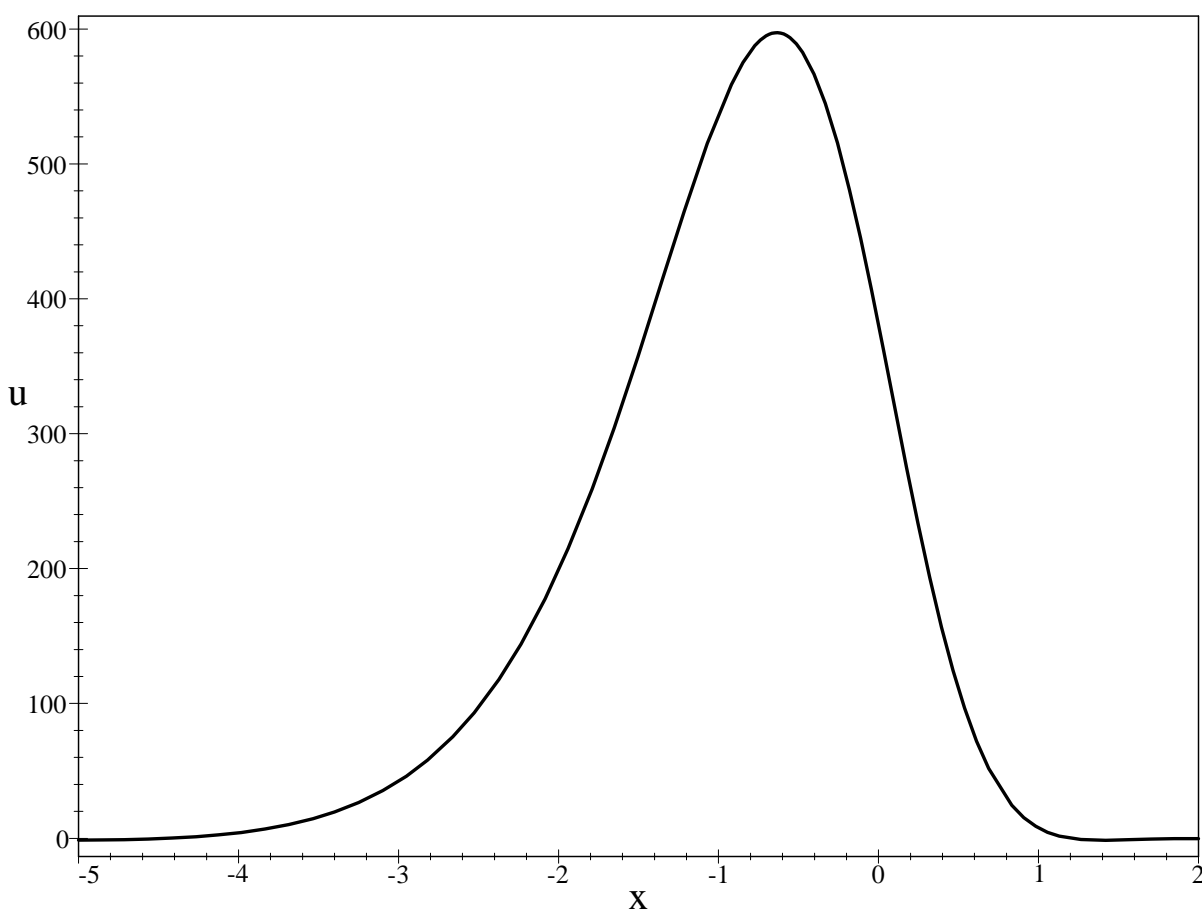

Fig. 1. Particular case of the solution (26) for the case when the solution of the simplest equation can be expressed by elementary functions. $t=0$. The values of the parameters are $\theta=1, \delta=2, \beta=2, \alpha=1, C^{*}=1, \gamma=1, \nu=1 . b_{2}=1.5, b_{3}=0.666173$. 


\section{Kink}

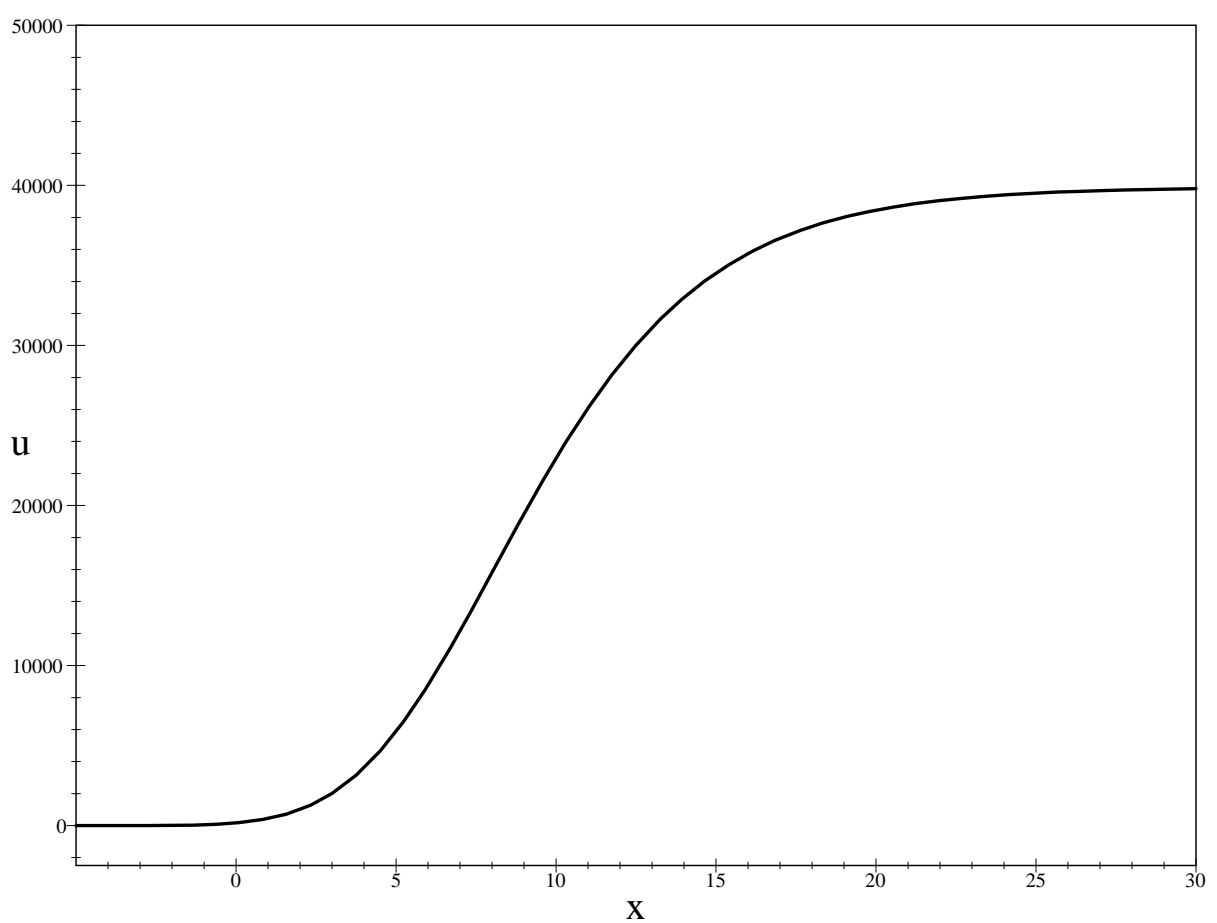

Fig. 2. Particular case of the solution (26) for the case when the solution of the simplest equation can be expressed by elementary functions. $t=0$. The values of the parameters are $\theta=1, \delta=2, \beta=2, \alpha=1, C^{*}=1, \gamma=1, \nu=1 . b_{2}=1, b_{3}=2.347300$.

keeping constant values of the parameters $\alpha, \beta, \gamma, \delta$ of the Kawahara equation. The parameters of the Abel equation $b_{2}$ and $b_{3}$ are varied and this variation leads to change of the kinds of the solution from a solitary wave to a kink.

\section{CONCLUding REMARKS}

In this paper we obtain exact traveling wave solutions of two nonlinear PDEs from the class (4) of generalized Kawahara equation. We present only particular analysis of the obtained solution of the generalized Kawahara equation, i.e., we show some forms of the solution only for the case $n=1$ and for the values of parameters for which the solutions are a solitary wave or a kink. Extensive analysis of the obtained solutions can be made by means of variation of larger number of parameters and by means of numerical investigation of the solutions for general case when they are expressed by the special function $V$. In addition an application of the obtained solutions for 
modeling shallow water waves in different systems can be made. All these topics will be discussed elsewhere.

\section{REFERENCES}

[1] N.K. Vitanov, Z.I. Dimitrova, K.N. Vitanov (2015) Modified Method of Simplest Equation for Obtaining Exact Analytical Solutions of Nonlinear Partial Differential Equations: Further Development of the Methodology with Applications. Applied Mathematics and Computation 269 363-378.

[2] M. Ablowitz, P.A. Clarkson (1991) "Solitons, Nonlinear Evolution Equations and Inverse Scattering". Cambridge University Press, Cambridge, UK.

[3] L. Debnath (2012) "Nonlinear Partial Differential Equations for Scientists and Engineers". Springer, New York, USA.

[4] A.W. Leung (1989) "Systems of Nonlinear Partial Differential Equations. Applications to Biology and Engineering". Kluwer, Dordrecht, Netherland.

[5] G.R. Gavalas (1968) "Nonlinear Differential Equations of Chemically Reacting Systems". Springer, New York, USA.

[6] J.D. MurRay (1977) "Lectures on Nonlinear Differential Equation Models in Biology". Oxford University Press, Oxford, UK.

[7] O. Nitcheva, B. Milev, T. Trenkova, N. Philipova, P. Dobreva (2018) Kamchia Watershed Groundwater Recharge Assessment by the CLM3 Model. MATEC Web of Conferences 14503011.

[8] O. Nitcheva (2018) Hydrology Models Approach to Estimation of the Groundwater Recharge: Case Study in the Bulgarian Danube Watershed. Environmental Earth Sciences $\mathbf{7 7} 464$.

[9] F. Verhulst (1990) "Nonlinear Differential Equations and Dynamical Systems". Springer, Berlin, Germany.

[10] N.K. Vitanov (2016) "Science Dynamics and Research Production. Indicators, Indexes, Statistical Laws and Mathematical Models". Springer, Cham, Switzerland.

[11] N.K. Vitanov, K.N. Vitanov (2016) Box Model of Migration Channels, Mathematical Social Sciences 80 108-114.

[12] M.J. Ablowitz, D.J. Kaup, A.C. Newell (1973) Nonlinear Evolution Equations of Physical Significance. Physical Review Letters 31 125-127.

[13] M.J. Ablowitz, D.J. Kaup, A.C. Newell, H. Segur (1974) Inverse Scattering Transform - Fourier Analysis for Nonlinear Problems. Studies in Applied Mathematics 53 249-315.

[14] C.S. Gardner, J.M. Greene, M.D. Kruskal, R.R. Miura (1967) Method for Solving Korteweg-de Vries Equation. Physical Review Letters 19 1095-1097.

[15] P. Holmes, J.L. Lumley, G. Berkooz (1996) "Turbulence, Coherent Structures, Dynamical Systems and Symmetry". Cambridge University Press, Cambridge, UK.

[16] E. Infeld, G. Rowlands (1990) "Nonlinear Waves, Solitons and Chaos". Cambridge University Press, Cambridge, UK. 
[17] N.A. KudRYAShov (1990) Exact Solutions of the Generalized Kuramoto-Sivashinsky Equation. Physics Letters A 147 287-291.

[18] A.C. Sсотт (1999) "Nonlinear Science. Emergence and Dynamics of Coherent Structures". Oxford University Press, Oxford, UK.

[19] A.C. Scotт (2002) "Neuroscience: A Mathematical Primer". Springer, New York, USA.

[20] M. TABOR (1989) “Chaos and Integrability in Dynamical Systems”. Wiley, New York, USA.

[21] N.K. Vitanov, I.P. Jordanov, Z.I. Dimitrova (2009) On Nonlinear Dynamics of Interacting Populations: Coupled Kink Waves in a System of Two Populations. Communications in Nonlinear Science and Numerical Simulation 14 2379-2388.

[22] N.K. Vitanov, I.P. Jordanov, Z.I. Dimitrova (2009) On Nonlinear Population Waves. Applied Mathematics and Computation 215 2950-2964.

[23] R. Hirota (1971) Exact Solution of Korteweg-de Vries Equation for Multiple Collisions of Solitons. Physical Review Letters 27 1192-1194.

[24] M. Remoissenet (1993) "Waves Called Solitons". Springer, Berlin, Germany.

[25] E. FAN, Y.C. Hon (2003) A Series of Travelling Wave Solutions for Two Variant Boussinesq Equations in Shallow Water Waves. Chaos, Solitons \& Fractals 15 559566.

[26] J.-H. HE, X.-H. Wu (2006) Exp-Function Method for Nonlinear Wave Equations. Chaos, Solitons \& Fractals 30 700-708.

[27] W. Malfliet, W. Hereman (1996) The tanh Method: I. Exact Solutions of Nonlinear Evolution and Wave Equations. Physica Scripta 54 563-568.

[28] A.M. WAZWAZ (2004) The tanh Method for Traveling Wave Solutions of Nonlinear Equations. Applied Mathematics and Computation 154 713-723.

[29] A.M. WAZWAZ (2009) "Partial Differential Equations and Solitary Waves Theory". Springer, Dordrecht, Netherlands.

[30] N.A. Kudryashov (2005) Simplest Equation Method to Look for Exact Solutions of Nonlinear Differential Equations. Chaos Solitons \& Fractals 24 1217-1231.

[31] N.A. Kudryashov, N.B. Loguinova (2008) Extended Simplest Equation Method for Nonlinear Differential Equations. Applied Mathematics and Computation 205 396402 .

[32] N.A. Kudryashov (2008) Solitary and Periodic Wave Solutions of Generalized Kuramoto-Sivashinsky Equation. Regular and Chaotic Dynamics 13 234-238.

[33] N.K. Vitanov, Z.I. Dimitrova, H. Kantz (2010) Modified Method of Simplest Equation and Its Application to Nonlinear PDEs. Applied Mathematics and Computation 216 2587-2595.

[34] N.K. Vitanov (2011) Modified Method of Simplest Equation: Powerful Tool for Obtaining Exact and Approximate Traveling-Wave Solutions of Nonlinear PDEs. Communications in Nonlinear Science and Numerical Simulation 16 1176-1185. 
[35] N.A. Kudryashov (2005) Exact Solitary Waves of the Fisher Equation. Physics Letters A 342 99-106.

[36] N.A. Kudryashov, M.V. Demina (2007) Polygons of Differential Equations for Finding Exact Solutions. Chaos Solitons \& Fractals 33 480-496. .

[37] N.A. KudRYASHOv (2010) Meromorphic Solutions of Nonlinear Ordinary Differential Equations. Communications in Nonlinear Science and Numerical Simulation 15 27782790.

[38] N.K. Vitanov (2010) Application of Simplest Equations of Bernoulli and Riccati Kind for Obtaining Exact Traveling Wave Solutions for a Class of PDEs with Polynomial Nonlinearity. Communications in Nonlinear Science and Numerical Simulation 15 2050-2060.

[39] N.K. Vitanov, Z.I. Dimitrova (2010) Application of the Method of Simplest Equation for Obtaining Exact Traveling-Wave Solutions for Two Classes of Model PDEs from Ecology and Population Dynamics. Communications in Nonlinear Science and Numerical Simulation 15 2836-2845.

[40] N.K. Vitanov, Z.I. Dimitrova, K.N. Vitanov (2011) On the Class of Nonlinear PDEs That Can Be Treated by the Modified Method of Simplest Equation. Application to Generalized Degasperis-Processi Equation and b-Equation. Communications in Nonlinear Science and Numerical Simulation 16 3033-3044.

[41] N.K. Vitanov, Z.I. Dimitrova, H. Kantz (2013) Application of the Method of Simplest Equation for Obtaining Exact Traveling-Wave Solutions for the Extended Korteweg-de Vries Equation and Generalized Camassa-Holm Equation. Applied Mathematics and Computation 219 7480-7492.

[42] N.K. Vitanov, Z.I. Dimitrova, K.N. Vitanov (2013) Traveling Waves and Statistical Distributions Connected to Systems of Interacting Populations. Computers \& Mathematics with Applications 66 1666-1684.

[43] N.K. Vitanov, Z.I. Dimitrova (2014) Solitary Wave Solutions for Nonlinear Partial Differential Equations That Contain Monomials of Odd and Even Grades with Respect to Participating Derivatives. Applied Mathematics and Computation 247 213-217.

[44] N.K. Vitanov (2019) Recent Developments of the Methodology of the Modified Method of Simplest Equation with Application. Pliska Studia Mathematica 30 29-42.

[45] N.K. Vitanov (2019) Modified Method of Simplest Equation for Obtaining Exact Solutions of Nonlinear Partial Differential Equations: History, Recent Developments of the Methodology and Studied Classes of Equations. Journal of Theoretical and Applied Mechnanics, Sofia 49 105-1XXX.

[46] E.V. Nikolova, I.P. Jordanov, Z.I. Dimitrova, N.K. Vitanov (2017) Evolution of Nonlinear Waves in a Blood-Filled Artery with an Aneurysm. AIP Conference Proceedings 1895070002.

[47] E.V. Nikolova, I.P. Jordanov, Z.I. Dimitrova, N.K. Vitanov (2018) Nonlinear Evolution Equation for Propagation of Waves in an Artery with an Aneurysm: An Exact Solution Obtained by the Modified Method of Simplest Equation. Studies in Computational Intelligence 728 131-144. 
[48] E.V. Nikolova, V.K. Kotev, G.S. Nikolova (2018) An Evolution Equation of Blood Flow in a Dilated Artery. IFMBE Proceedings 65 209-2013.

[49] E.V. Nikolova (2018) On Nonlinear Waves in a Blood-Filled Artery with an Aneurysm. AIP Conference Proceedings 1978470050.

[50] E.V. Nikolova (2019) Evolution Equation for Propagation of Blood Pressure Waves in an Artery with an Aneurysm, Studies in Computational Intelligence 793 327-339.

[51] N.K. Vitanov, Z.I. Dimitrova (2018) Modified Method of Simplest Equation and the Nonlinear Schrödinger Equation. Journal of Theoretical and Applied Mechanics, Sofia 48 (1) 59-68.

[52] N. Martinov, N. Vitanov (1992) On Some Solutions of the Two-Dimensional sineGordon Equation. Journal of Physics A: Mathematical and General 25 L419-L425.

[53] N. Martinov, N. Vitanov (1992) Running Wave Solutions of the Two-Dimensional sine-Gordon Equation. Journal of Physics A: Mathematical and General 25 3609-3613.

[54] N.K. Martinov, N.K. Vitanov (1994) New class of Running-Wave Solutions of the (2+1)-Dimensional sine-Gordon Equation. Journal of Physics A: Mathematical and General 27 4611-4618.

[55] N.K. Vitanov (2011) On Modified Method of Simplest Equation for Obtaining Exact and Approximate Solutions of Nonlinear PDEs: the Role of the Simplest Equation. Communications in Nonlinear Science and Numerical Simulation 16 4215-4231.

[56] T. KaWAHARA (1972) Oscillatory Solitary Waves in Dispersive Media. Journal of the Physical Society of Japan 33 260-264.

[57] M.L. Gandarias, M. RosA, E. Recio, S. Anco (2017) Conservation Laws and Symmetries of a Generalized Kawahara Equation. AIP Conference Proceedings 1836 020072.

[58] A. BiswAS (2009) Solitary Wave Solution for the Generalized Kawahara Equation. Applied Mathematical Letters 22 208-210.

[59] R. Grimshaw, B. Malomed, I. Benilov (1994) Solitary Waves with Damped Oscillatory Tails: an Analysis of the Fifth-Order Korteweg-de Vries Equation. Physica D 77 473-485.

[60] P. Dias, P. Milewski (2010) On the Fully Nonlinear Shallow-Water Generalized Serre Equations. Physics Letters A 374 1049-1053.

[61] A.T. Ilichev, V.Y. TomaspolskiI (2015) Soliton-Like Structures on a Liquid Surface under an Ice Cover. Theoretical Mathematical Physics 182 231-245. 